\title{
Correction to: Ab Initio Calculated Results Require New Formulations for Properties in the Limit of Zero Density: The Viscosity of Methane $\left(\mathrm{CH}_{4}\right)$
}

\author{
Arno Laesecke ${ }^{1}$ • Chris D. Muzny ${ }^{1}$
}

Published online: 13 March 2018

(C) Springer Science+Business Media, LLC, part of Springer Nature 2018

\section{Correction to: Int J Thermophys (2017) 38:182 https://doi.org/10.1007/s10765-017-2305-8}

The authors would like to correct a misplaced parenthesis in the equation of their Correction published to correct the error in Eq. 3 of the original version (Correction https://doi.org/10.1007/s10765-017-2355-y). Dr. Robert Hellmann of the University of Rostock (Germany) has advised that the correct viscosities for methane are obtained with the equation given in the Correction when the last parenthesis in the denominator is moved left to close the denominator of parameter $a_{1}$

$$
\eta_{0}(T)=\frac{C \sqrt{T}}{a_{0}+a_{1} /\left(T^{1 / 6} \exp \left(T^{1 / 6}\right)\right)+a_{2} T^{1 / 6}+\left(a_{3}+a_{4} T^{1 / 6}\right) /\left(a_{5}+T^{1 / 6} \exp \left(a_{6} / T^{1 / 6}\right)\right)} .
$$

This formula is identical to Eq. 3 in the original paper except for the mathematical operations associated with parameters $a_{2}$ and $a_{6}$. The authors apologize for the confusion that their errors have caused and thank Dr. Hellmann for bringing this typesetting error to their attention. None of the other results or conclusions of the work are affected by this correction. The authors will submit future contributions directly to the Journal of Irreproducible Results.

The original article can be found online at https://doi.org/10.1007/s10765-017-2305-8.

Arno Laesecke

Arno.Laesecke@Boulder.NIST.Gov

1 Material Measurement Laboratory Applied Chemicals and Materials Division, National Institute of Standards and Technology, 325 Broadway, Boulder, CO 80305-3337, USA 